\title{
XXVII. On some physical relations affecting matter in diverse stages of subdivision
}

\section{Dr. S. Tolver Preston}

To cite this article: Dr. S. Tolver Preston (1908) XXVII. On some physical relations affecting matter in diverse stages of subdivision , Philosophical Magazine Series 6, 16:92, 345-351, DOI: 10.1080/14786440808636514

To link to this article: http://dx.doi.org/10.1080/14786440808636514

Published online: 21 Apr 2009.

Submit your article to this journal $₫$

Џ Article views: 3

Q View related articles $\square$ 
I do not know the exact ratio between the numbers of turns on the primary and on the secondary of this inductioncoil, but it is probably of the order of 100 , so that the 35 milliamperes flowing in the negative direction in the secondary would correspond to a demagnetizing current of about $3 \frac{1}{2}$ amperes in the primary, which would leave a comparatively small margin of resultant magnetizing ampere-turns.

If this is the case, the energy that can be got out of the secondary on break is very limited, which would account for the rapid dying away of the current to zero when even a very small spark-gap is introduced in the circuit. Directly the length of the spark-gap is sufficient to prevent the current flowing in the negative direction round the secondary circuit, the whole of the demagnetizing effect of the secondary current is done away with, and under the conditions of the experiment the magnetizing current in the primary is mainly limited by the large resistance in the circuit ; hence, we get a very much larger amount of energy available directly the secondary current is prevented from flowing in the negative direction. This I think accounts for the large difference in the size of the current waves in fig. 6 , and shows the great importance of preventing any current from flowing round the secondary circuit in the negative direction at make when using the induction-coil on a high voltage supply.

I made certain that the phenomenon was not due to any want of symmetry in the points of the spark-gap by reversing the electrodes of the gap and also by interchanging the connexions to it. The material of the electrodes did not seem to appreciably affect the results, but the shape of the electrodes was important in so far that the spark-length at which the galvanometer deflexion changed sign depended on the shape. Thus with two spheres the galvanometer deflexion changed sign at a shorter length than with points.

I have brought these two observations forward in the hope that in the discussion either my views as to the explanations may be confirmed or that better ones may be suggested.

XXVII. On some Physical Relations affecting Matter in Diverse Stages of Subdivision. By Dr. S. Tolver Preston*.

THE phrase "Continuity" is employed here in respect to 1 certain physical qualities, in actual practice as efficacious as if continuity in an absolute sense existed. We refer, for instance, to the capacity to maintain a sensibly equable * Communicated by the Author.

Phil. Mag. S. 6. Vol. 16. No. 92. Aug. 1908. 
pressure about a small object (say a sphere ${ }^{*}$ of finite radius) by the simplest kinetic medium composed of particles of matter or "corpuscules" in a normal state of motion. The absence of a universal agreement as to the ultimate constitution of Matter, does not prevent the deduction of certain physical relations rigorously resulting from given premisses.

The more the material constituting our physical agent (sometimes termed "medium") diffused in space is subdivided, the more equable evidently will the pressure (kinetic) about the said finite small sphere become, until, in the limit, by progressive subdivision of the material, the pressure comes to rival in steadiness that of a perfectly continuous fluid. Yet, at the same time, the medium might represent a Vacuity far surpassing that of any "Vacuum" artificially obtainable, in regard to relatively small proportion of material contained in the medium, compared with the total (pervaded) space.

In this way, by simple subdivision, a single molecule of gross matter might be imagined capable of pervading the range of the visible Cosmos, - with minute "corpuscules" in close proximity (reckoned from centre to centre). It may possibly be well to realize that there is no assignable limit (practically speaking) to this procedure, in conception at least : and thus dimensions actually measured or calculated may possibly be easier brought within mental grasp, in connexion with the Corpuscular view of Matter.

"(Oontinuity" may (additionally) refer to that sensible physical continuity, in reference to the impossibility of directly detecting any trace of structure anywhere in our medium, on account of its dimensional properties eluding direct appreciation by the senses; so that the appearance is presented of the continuity of a fluid. Thus the extremes of Vacuity and of Continuity may be said "to meet," -in a certain physical signification.

That the " mean length of path," by this process of subdivision of material, also diminishes continuously in the same

* Given a normal velocity for the constituent particles, the sensible uniformity of the pressure of a medium [say our atmosphere] upon a small body immersed therein, evidently does not depend on the size of the impinging air-particles, which maintain the pressure, but on their proximity (from centre to centre), or on their number in unit of volume. Hence, by simple subdivision of the material, we observe that any degree of Continuity combined with Vacuity [i.e., optionally small collective volume of material compared with the total volume of space pervaded]_may be practically achieved, capable of serving useful ends. The so-termed "small " body evidently must not be so reduced that its dimensions become comparable to the mean distance [relatively inferior] of the component particles of the medium, in which it is located. 
ratio as the other dimensional elements, may not possibly appear so evident at first sight.

An ideal case, although not exactly applicable in nature, may, as approximately applicable, often be of practical utility to illustrate closely natural facts. Thus actually, by diverse stages of aggregation of material into grosser clusters, interstices exist between the component corpuscules which constitute the cluster, and that even if the component corpuscules be supposed to possess some elasticity [adaptation of shape to pressure], or imagined to be in actual contact. Our object here is to consider the ideal case where the interstices eliminate themselves.

The electron theory of the corpuscular constitution of Matter is taking a foremost place in philosophical thought in the present day. May it not be conceivable that in certain regions of the universe these corpuscules part with their motion (whether by transference \&c.) If so, then under central forces such as gravitation \&c. the corpuscules, if elastic, could aggregate into masses practically continuous [comparable to a continuous fluid], without interstices, excepting possibly near the exterior confines of the masses. The very high intensity of motion of corpuscules, observed by us, may well only exist in a relatively small proportion of the universe, namely in those (probably exceptional) regions where Life, as known to us, prevails.

While physical consequences or relations in their ideal exactness may of course have a permanence not unlike mathematical truths, they may at the same time apply to possible realities of Nature with sufficient approximation to merit that realization which makes knowledge productive.

Let us consider the in some respects ideal case of a perfect fluid in diverse stages of subdivision into spherical globules, and where, under assumed central forces, the globules [however small by subdivision, or however large, by aggregation, they may become] automatically resume the spherical form when left to themselves.

On the above premisses, we observe it to be readily demonstrable that a progressive subdivision of material to stages finer and finer, has the consequence that the radii of the (globular formed) corpuscules thus set free, their mean distance, reckoned from centre to centre, with their " mean length of path," - these dimensional properties (of the medium thus constituted), although changing absolutely, are relatively unalterable by this process of subdivision of the material and consequent multiplication of available parts, but remain as constant as the quantity of material itself. 


\section{Dr. S. Tolver Preston on some Physical Relations}

We may notice then that the "mean length of path" of an ideal stellar mass (generated by inverse aggregation out of corpuscules) would be the same multiple of the radius of the stellar mass, as the mean length of path of a corpuscule was (beforehand) of its radius. Nevertheless, in view of the familiar phrase "sparsely scattered stars," the first case would be considered as illustrative of Vacuity, while the second exemplifies the Continuity of some medium where the dimensional elements elude our direct powers of measurement. Yet in both cases, the dimensional elements (as we may note) may actually be similar.

By supposed progressive subdivision of the material under the conditions cited, we may observe the globular corpuscules thus set free gradually approaching each other (as to their geometrical centres) asymptotically, without assignable limit as to proximity :-all the dimensional properties of the medium thus constituted [including length of mean path] diminishing in a like proportion. The recognized Relativity of the results reached does not exclude the possibility of practical or useful consequences in connexion with the economy of Nature, which mental realization may go further to develop.

Elementary illustrative methods (wherever possible), may be not without their value. If we suppose a cubical element of space, with portions of material in spherical form situated at the eight corners of this cube, and, for the concept of ready subdivision into parts, suppose the material to be a continuous or perfect fluid, with a central force acting to maintain any portion thereof globular when set free : then it becomes evident (illustratively) that when by the subdividing process, the radius of a fluid globe is reduced to one half, eight times as many then become available ;-permitting eight times as many cubical space-elements to be furnished with portions of material at their corners. Then, by the simple subdivision of our material, (obviously) the side of each cubical space-element [representing the distance of the portions of material from centre to centre], becomes exactly halved.

Clearly, in this way, by a progressively finer stage of subdivision of material, we have reached an exactly similar configuration (geometrically), in regard to relative radii of the globules [corpuscules], relative distances, reckoned from centre to centre, \&c. as those with which we started:-this continuing to hold true if the process of subdivision of material were carried out without assigned limit.

Accordingly we may observe that whatever multiple of the 
radius of a globule [or globe] the mean length of path was at the commencement, it will remain that same multiple, however far the process [whether of subdivision or of inverse aggregation] be conceived to be carried. In some relational sense, in respect to small scale, we might even speak of an ætherial medium, or, in respect to big scale, of a stellar medium.

Somewhat in correlation with the present subject-in 'Nature,' September 28, 1893, p. 517, I ventured critically to comment a view of $\mathrm{M}$. Delbœuf, given in Bulletin de l'Académie de Belgique, No. 6, 1893, who contests Laplace's conclusion as to the Relativity of all dimensions, velocities, \&c., in regard to the visible universe. In the abstract of M. Delbouf's paper, Laplace's view is quoted as follows :-

"According to Laplace, if the dimensions of all the bodies in the universe, their mutual distances and velocities were to increase or diminish in a constant proportion, these bodies would describe the same curves as they do now. The appearances presented to observers would be the same, and independent of the dimensions assumed. Hence the only facts we are able to appreciate are ratios." ("Nature,' August 24, 1893, p. 406.)

It is added (in the abstract in 'Nature') that considerations pursued by $M$. Delbœuf into directions other than concerning dimensions alone, "go to show that real space is different from geometric space, and that the dimensions of the universe are absolute" (p. 406).

But surely Laplace must have implied that not only velocities, but forces \&c. were supposed to vary in correspondence, so as to maintain the previous state of equilibrium undisturbed. For if equilibrium were upset, there would be no fair test possible of the power of observation to detect a change of dimensions accomplished,-which is the point of debate.

In my communication to 'Nature' of the above date, under the same title as that chosen by M. Delbcuf-"Megamicros,"I offered as an illustrative case a human being grasping a metrically graduated staff equal in length to his own height.

By any supposed reduction in size, simultaneously with the dimensional scale of our universe (in extent conceived * radially finite), there would be nothing positive to indicate that a change of size had effected itself, while the metrical staff grasped by the observer would still be the same length as

* Without necessarily postulating any particular limit to the Cosmos, we may perfectly well suppose the above modifications to be confined to our universe, $i$. e. to the lnnown, within the range of telescopic vision, -leaving the unknown entirely ont of consideration. 
himself, - and that if dimensions oscillated between the infinites. We seem in this way to bridge the infinite (so to say) in extremes of thought.

To connect dimensions (if only in one isolated instance) with our idea of the indestrnctibility of Matter, we might imagine the metrical standard of the "Conservatoire" to have alone remained unaltered in length, when the accomplished reduction (or increase) of size of everything else would then become obvious,-by comparison with the metrical standard left over as the sole test-relic of the previous state of Relativity. Such modification of dimensions of our universe would evidently imply a proportional "creation" or (respectively) "annihilation" of Matter, with the concomitant shortening (or lengthening) of the radius of extension of the whole system affected. And if these changes of dimensions be imagined to be instantaneous, the conservation of the memory-mechanism (itself independent of scale, and united with a psychological side), would secure the unbroken recognition of undisturbed Relativity.

In order to reason effectively on some questions, we may fitly make certain preliminary assumptions, which do not abate the logic of the conclusions.

The catastrophic change of absolute size (by unaltered relative dimensions of internal constituents) of our cosmos, with variation of radius of extension, implied in the passage from one state of Relativity to another, - must be imagined for the purpose of our argument) to be perfectly instantaneous.

It appears, however, that the fact of a physical catastrophe being involved in such changes, goes to demonstrate that there is such a thing as absolute size, or that this phrase has a meaning within the domain of Physical Science. Indeed, in the absence of the concept of "absolute size," as a basis to rest on, it seems that the abstract idea of infinite extension oscillates hither and thither in uncertainty (as if its logical justification were in question).

Only "absolute size" appears to possess this peculiarity, that any imagined fluctuations of its value [in exact parallelism with that of the metrical standard-by the conservation of Relativity], would elude our observation, even under the implied retention of memory throughout. For the independence of the most elaborate mechanisms on absolute, and their unique dependence on relative scale of parts, - is an obvious and accepted fact in Engineering design.

Gross Flottbek, bei Altona, Germany,

Dec. 1907-April 1908. 
[Added June 11, 1908.] - It may be noticed that under the radial (central) action of its own gravitational forces, a mass of matter, by mere increase of size,-becomes elastic. The forcible recovery of the spherical form, under the radial stress, amounts substantially to a power of elastic restitution.

Let us suppose a nebula, instead of consisting of those smaller fragments of material to which we apply the name "meteorites," after disintegration in our atmosphere,-to be constituted of masses more comparable to the earth in size [small in relation to the solar dimensions]:- these energetic bodies automatically setting up that equilibriated movement among themselves characteristic of the (minuter) particles of a gas. Then such gravitationally-elastic portions of material constituting an attenuated nebula, and surviving without encounters for lengthy time-periods, would strive to conserve the natural globular form [vibrating about the same], conditioned by the powerful radial stress of gravity in each case: but coalescence into larger masses (spherical) now and then, by relaxation of the translatory motion, is of course not excluded.

An inert mass of (say) the solar diameter, if we suppose broken up by a quick encounter, would then, even if cold and solidified throughout, resolve itself into globular fragments, and not into portions of irregular shape,-excepting those splinters below a certain limit of size.

And this would doubtless be the essential primary stage of such a cosmic encounter :- a truly gaseous nebula demanding time to constitute itself. It appears, accordingly, that the translatory energy of the encounter would at first continue mainly as mass-motion :- passing more gradually into the finer motion of molecules.

XXVIII. Notices respecting New Books.

A Treatise on Ifydraulics. By Wildiam Cawthonne Unwin, LL.D., F.R.S. London : A. \& C. Black. 1907.

THIS book may in a sense be regarded as a new edition of the 1 valuable article contributed by Professor Unwin to the Encyclopadia Britannica more than thirty years ago. Certain paragraphs and many of the diagrams are simply reproduced, but the omission of much of the former detail and the substitution of more recent data, with a complete recasting of many essential parts, make the treatise an altogether new work. To one familiar with the article of 1867 , a glance through the book will reveal many similarities; but a careful inspection will show that there are many more differences, and that Professor Unwin has spared no pains to bring his work up to date. A complete chapter on the distri- 